\title{
Experimentally Verified Minimal Cardiovascular System Model For Rapid Diagnostic Assistance
}

Bram W. Smith ${ }^{1}$, J. Geoffrey Chase ${ }^{2}$, Geoffrey M. Shaw ${ }^{3}$ and Roger I. Nokes $^{4}$

Center for Model-based Medical Decision Support (MMDS)

\author{
Aalborg University \\ Fredrik Bajers Vej 7F \\ DK-9220 Aalborg East \\ Denmark
}

Email: bws@hst.auc.dk

Phone: +4596358764

Fax: +4596358764

\footnotetext{
${ }^{1}$ Centre for Model-based Medical Decision Support, Aalborg University, Aalborg, Denmark

${ }^{2}$ Centre for Bio-Engineering, Department of Mechanical Engineering, University of Canterbury, Christchurch, New Zealand

${ }^{3}$ Centre for Bio-Engineering, Christchurch Hospital Dept of Intensive Care, Christchurch, New Zealand

${ }^{4}$ Dept of Civil Engineering, University of Canterbury, Christchurch, New Zealand
} 


\begin{abstract}
Characterising circulatory dysfunction and choosing a suitable treatment is often difficult and time consuming. This paper outlines a numerically stable minimal model of the human cardiovascular system (CVS) specifically aimed for rapid, on site modelling to assist in diagnosis and treatment. A minimal number of governing equations and a realistic valve law are used to accurately capture trends in CVS dynamics. The model is shown to have long-term stability and consistency with non-specific initial conditions. Examples of model verification are shown for experimentally measured static and transient response data. The model is also verified to capture commonly seen changes in CVS function as a result of disease. These examples illustrate the power of the minimal model for capturing CVS dynamics in health and disease, while its simplicity enables its use as a clinical aid.

Keywords: Biomedical control, Biomedical systems, Non-linear models, Physiological models, Cardiovascular Models, Circulation Models, Heart models
\end{abstract}

\title{
1 Introduction
}

Statistics from the American Heart Association (2004) show cardiovascular disease accounted for $38.5 \%$ of all deaths in America in 2001. Often it is difficult for health professionals to diagnose and select a suitable treatment for these conditions, and they must rely solely on their experience and intuition. For patients in a critical condition, under intensive care, the longer it takes to diagnose and treat a problem the more the condition can deteriorate, and 
the lower the chance of recovery. The goal of this research is to develop a model of the human cardiovascular system simple enough to assist medical staff in the key areas of understanding, rapid diagnosis and therapy selection.

With an appropriate model, medical staff can gain a better understanding of CVS function by varying parameters to simulate a variety of dysfunctions, such as stiff heart walls or blocked arteries. A model whose parameters have been identified to match a specific patient could be used to assist diagnosis and treatment. By measuring various physiological variables such as pressures, flow rates and heart rate, the governing elastances, resistances and pressure-volume (PV) relationships for a given patient's haemodynamics can be determined. These results can be gained quickly to allow immediate feedback to medical staff if the model complexity is minimised. Identification of patient specific parameters would enable detection of irregularities and the simulated testing of several potential therapies.

It is intended that the model fulfil the following aims to ensure that it is practical and effective as a diagnostic aid:

- Model parameters can be relatively easily identified for a specific patient.

- Although quantitatively exact results are not necessary, accurate prediction of trends with changes in parameters or therapy is required.

Most current approaches to modelling the human CVS can be grouped into either Finite Element (FE) or Pressure Volume (PV) approaches. FE techniques offer accurate results, but require immensely detailed inputs such as muscle fibre orientations, structures and mechanical properties [Peskin 
and McQueen, 1992; Legrice et al., 1997]. Limitations on the availability of detailed patient specific data and computational power mean that FE methods are not well suited as rapid diagnostic tools.

PV methods divide the CVS into a series of elastic chambers separated by resistances simulating pressure drops, and inductors simulating inertial effects where required. Each elastic chamber models a section such as the ventricles, the atria, or the aorta, each with their own pressure-volume relationship. Only a minimal number of parameters, such as chamber elastances and arterial resistances, are required to create such a model. These models can be solved on modern, commonly available desktop computers in reasonable time suitable for immediate clinical feedback.

This paper outlines a stable "Minimal Model" approach to the CVS modelling problem using a minimal number of governing equations. Problems with long-term divergence and dependence on accurate initial conditions, caused by over-defining the model, were encountered with other similar models (e.g. Chung et al., (1997)). The minimal model is developed starting with a simple single ventricle system and progressively adding complexity. The emphasis is on using the minimal amount of parameters and complexity required to model the essential dynamics and trends.

Model verification is then performed by comparing model outputs with published trends for both static and transient CVS dynamics in health and disease. The examples shown in this paper summarise the methods used to verify the model which are covered in much more detail in Smith (2004). The aim is to assess the model's capability to capture these trends before more detailed experimental research and validation is carried out in the hospital 
environment.

\section{Model Structure and Definition}

The model presented is intended to simulate the cardiovascular system, which is made up of the heart, and the pulmonary and systemic circulation systems, as shown in Figure 1. The full model presented is shown in Figure 2, where two ventricles simulate the heart. Two additional chambers are used for blood flow through the pulmonary circulation, in the lungs, and through the systemic circulation, in the rest of the body. The atria have not been added as they contribute only minimally to the main cardiac trends and can be easily added for more specific cases. The ventricles are coupled to account for important ventricular interaction dynamics due to both the common wall between the ventricles, called the septum, and outer wall of the heart, called the pericardium.

In Figure 2, resistances $(\mathrm{R})$ simulate the resistance on the blood passing through the arteries or valves between elastic chambers. Inductors (L) model the effects of inertia, which are only included where the blood goes through large changes in velocity. Thus, inertia is included around the valves where velocity pulses between zero and a peak value [Melchior et al., 1992; Smith et al., 2003; Smith, 2004]. Diodes show the location of the one-way valves at the inlet and exit of the ventricles. 


\subsection{Single Chamber Model}

The single elastic chamber shown in Figure 3 was analysed first to understand the dynamics of a single active chamber such as a ventricle. This model is similar to the Windkessel circuits in the literature, but with a simple elastic chamber rather than a capacitor (e.g. Tsitlik et al., (1992)). Resistors (R) and inductors (L) at the inlet and outlet of the chamber model the resistance and inertial effects on flow entering or exiting the chamber.

\subsection{The PV Diagram}

PV diagrams, such as the one shown in Figure 4, are used extensively in the medical profession. Importantly, doctors are familiar with, and comfortable using, PV diagrams to analyse ventricular function, as evidenced by there regular use in clinical textbooks [Guyton and Hall, 2001; Braunwald et al., 2001]. The two main characteristics of the PV diagram are the lines plotting the End Systolic Pressure-Volume Relationship (ESPVR) and the End Diastolic Pressure-Volume Relationship (EDPVR). The EDPVR represents the minimum elastance of the ventricle during diastole when the ventricle is filling. The ESPVR represents the maximum elastance of the ventricle at the end of the systole, or ventricle ejection. These lines define the upper and lower limits respectively of time varying ventricular elastance during the cardiac cycle.

Equations approximating the ESPVR and EDPVR lines are widespread throughout the literature [Maughan et al., 1987; Chung et al., 1997; Beyar et al., 1987]. The most commonly used relationships are defined below 
[Chung et al., 1997; Beyar et al., 1987]:

$$
\begin{gathered}
\mathrm{P}_{\text {es }}(\mathrm{V})=\mathrm{E}_{\mathrm{es}}\left(\mathrm{V}-\mathrm{V}_{\mathrm{d}}\right) \\
\mathrm{P}_{\text {ed }}(\mathrm{V})=\mathrm{P}_{0}\left(\mathrm{e}^{\lambda\left(\mathrm{V}-\mathrm{V}_{0}\right)}-1\right)
\end{gathered}
$$

where Equation (1) is the linear relationship between the end systolic pressure $\left(\mathrm{P}_{\mathrm{es}}\right)$ and volume $(\mathrm{V})$ with elastance $\left(\mathrm{E}_{\mathrm{es}}\right)$ and intercept $\left(\mathrm{V}_{\mathrm{d}}\right)$. Equation (2) plots the non-linear relationship between end diastolic pressure $\left(\mathrm{P}_{\mathrm{ed}}\right)$ and volume $(\mathrm{V})$ with the additional parameters $\mathrm{P}_{0}, \lambda$, and $\mathrm{V}_{0}$ which define the EDPVR curve. $\mathrm{V}_{0}$ defines the EDPVR volume at zero pressure, $\mathrm{P}_{0}$ defines the elastance of the ventricle and $\lambda$ defines the curvature of the EDPVR line.

\subsection{Cardiac Driving Function}

The contractions of the cardiac muscle are modelled by a time varying chamber elastance that varies between a minimum value defined by the EDPVR and a maximum value defined by the ESPVR. A driving function, e(t), that varies approximately sinusoidally between 0 and 1 drives the changes in elastance to make the heart pump [Chung et al., 1997]. Combining the driver definition with Equations (1) and (2) produces an equation for the chamber pressure $\left(\mathrm{P}_{2}\right)$ :

$$
\begin{gathered}
\mathrm{P}_{2}(\mathrm{~V}, \mathrm{t})=\mathrm{e}(\mathrm{t}) \mathrm{P}_{\text {es }}(\mathrm{V})+(1-\mathrm{e}(\mathrm{t})) \mathrm{P}_{\text {ed }}(\mathrm{V}) \\
\mathrm{e}(\mathrm{t})=\sum_{\mathrm{i}=1}^{\mathrm{N}} \mathrm{A}_{\mathrm{i}} \mathrm{e}^{-\mathrm{B}_{\mathrm{i}}\left(\mathrm{t}-\mathrm{C}_{\mathrm{i}}\right)^{2}}
\end{gathered}
$$


Equation (3) is now the governing equation for the time dependent pressure volume relationship in an active elastic chamber such as a ventricle. Equation (4) defines the driver function used here with parameters $N=1, A=1$, $\mathrm{B}=80$ secs $^{-2}$ and $\mathrm{C}=0.27$ secs used to define the function's profile.

\subsection{Fluid Flow Rate Definitions}

When the effects of inertia are not included, the equation for the flow rate (Q) between two pressures $\left(\mathrm{P}_{1}\right.$ and $\left.\mathrm{P}_{2}\right)$ across resistance $\left(\mathrm{R}_{1}\right)$, as defined in Figure 3, is Poiseuille's equation [Fung, 1990; Beyar et al., 1987; Chung et al., 1997; Olansen et al., 2000].

$$
\mathrm{Q}_{1}=\frac{\mathrm{P}_{1}-\mathrm{P}_{2}}{\mathrm{R}_{1}}
$$

where the resistance $(\mathrm{R})$ is defined by Poiseuille's equation as $\mathrm{R}=\pi \mathrm{r}_{0}^{4} / 8 \mu \ell$ with variables for radius $\left(\mathrm{r}_{0}\right)$, fluid viscosity $(\mu)$, and artery length $(\ell)$.

Equation (5) allows discontinuous changes in flow rate, meaning the flow rate can change instantaneously, ignoring inertial effects on the fluid. When inertial effects are added, the equation of motion for the flow can be approximated as the differential equation [Smith et al., 2003; Smith, 2004]:

$$
\frac{\mathrm{dQ}}{\mathrm{dt}}=\frac{\mathrm{P}_{1}-\mathrm{P}_{2}-\mathrm{Q}_{1} \mathrm{R}_{1}}{\mathrm{~L}_{1}}
$$

With the governing equations for flow rate, the rate of change of volume in the chamber is simply calculated as the inflow minus the outflow:

$$
\frac{\mathrm{dV}}{\mathrm{dt}}=\mathrm{Q}_{\text {in }}-\mathrm{Q}_{\text {out }}
$$


Thus, the dynamics of a single ventricle model with resistances and inertial effects are defined by Equations (6) and (7). Given volume (V) and time (t), the pressure in the chamber $\left(\mathrm{P}_{2}(\mathrm{~V}, \mathrm{t})\right)$ can be calculated using Equation (3). The flow rates are then calculated using either Equation (5) or Equation (6), and from the flow rate, the rate of change of volume is calculated using Equation (7).

\subsection{Two Ventricle Model with Interaction}

Ventricular interaction has a significant impact on cardiovascular dynamics, and is caused by both the septum and the pericardium. The double lines between the left and right ventricles in the full model of Figure 2 indicate the coupling due to ventricular interaction. Figure 5 shows the cardiac geometry employed, and defines the necessary volume and pressure variables.

The volumes shown in Figure 5 include the 2 ventricle volumes, and 3 free wall volumes of the ventricles and septum. The free wall volumes, $V_{\text {lvf }}, V_{\text {rvf }}$ and $V_{\text {spt }}$, are not chamber volumes, but are defined to capture the deflection of the cardiac free walls relative to the ventricle volumes.

The left ventricle free wall volume from Figure 5 is simply the left ventricle volume less the septal volume. Similarly, the right ventricle free wall volume is the sum of the right ventricle volume, and the septum volume.

$$
\begin{gathered}
\mathrm{V}_{\mathrm{lvf}}=\mathrm{V}_{\mathrm{lv}}-\mathrm{V}_{\mathrm{spt}} \\
\mathrm{V}_{\mathrm{rvf}}=\mathrm{V}_{\mathrm{rv}}+\mathrm{V}_{\mathrm{spt}}
\end{gathered}
$$


The pericardium volume is the sum of the ventricle volumes, or the sum of the ventricle free wall volumes.

$$
\mathrm{V}_{\mathrm{pcd}}=\mathrm{V}_{\mathrm{lv}}+\mathrm{V}_{\mathrm{rv}}=\mathrm{V}_{\mathrm{lvf}}+\mathrm{V}_{\mathrm{rvf}}
$$

The total volume of the pericardium defined in this model excludes the volume of the atria.

The pressures defined for the two-ventricle model and shown schematically in Figure 5. The left and right ventricle and pericardium chamber pressures are defined:

$$
\begin{gathered}
\mathrm{P}_{\mathrm{lv}}=\mathrm{P}_{\mathrm{lvf}}+\mathrm{P}_{\text {peri }} \\
\mathrm{P}_{\mathrm{rv}}=\mathrm{P}_{\mathrm{rvf}}+\mathrm{P}_{\text {peri }} \\
\mathrm{P}_{\text {peri }}=\mathrm{P}_{\mathrm{pcd}}+\mathrm{P}_{\mathrm{th}}
\end{gathered}
$$

The septum pressure, using Equations (11) and (12), is therefore defined:

$$
\mathrm{P}_{\mathrm{spt}}=\mathrm{P}_{\mathrm{lv}}-\mathrm{P}_{\mathrm{rv}}=\mathrm{P}_{\mathrm{lvf}}-\mathrm{P}_{\mathrm{rvf}}
$$

Contraction and relaxation of the heart occurs as a result of free wall thickening. Hence, the left and right ventricle and septal free wall pressures $\left(\mathrm{P}_{\text {lvf }}, \mathrm{P}_{\mathrm{rvf}}\right.$ and $\left.\mathrm{P}_{\mathrm{spt}}\right)$ are calculated using Equation (3). For example the pressure in the left ventricle free wall is given as:

$$
\mathrm{P}_{\text {lvf }}=\mathrm{e}(\mathrm{t}) \mathrm{E}_{\mathrm{es}, \mathrm{lvf}}\left(\mathrm{V}_{\mathrm{lvf}}-\mathrm{V}_{\mathrm{d}, \mathrm{lvf}}\right)+(1-\mathrm{e}(\mathrm{t})) \mathrm{P}_{0, \mathrm{lvf}}\left(\mathrm{e}^{\lambda_{\mathrm{lvf}}\left(\mathrm{V}_{\mathrm{lvf}}-\mathrm{V}_{0, \mathrm{lvf}}\right)}-1\right)
$$


Finally, the pressure across the passive wall of the pericardium is defined by the nonlinear PV relationship [Chung et al., 1997]:

$$
\mathrm{P}_{\mathrm{pcd}}\left(\mathrm{V}_{\mathrm{pcd}}\right)=\mathrm{P}_{0, \mathrm{pcd}}\left(\mathrm{e}^{\lambda_{\mathrm{pcd}}\left(\mathrm{V}_{\mathrm{pcd}}-\mathrm{V}_{0, \mathrm{pcd}}\right)}-1\right)
$$

The pressures calculated using these equations are used to calculate the flow rates into and out of the cardiac chambers.

\subsection{Full Closed System Loop Model}

To capture more of the major governing haemodynamics, the circulation must be closed so that fluid flows around the entire loop, as in Figure 2. Two elastic chambers are added for each of the systemic and pulmonary systems with a resistance between. Each chamber is modelled using the linear PV relationship in Equation (1). With the output of each cardiac chamber connected to the inlet of the other, peripheral elastances and resistances capture the basic dynamics of the pulmonary and systemic circulation.

\section{Dynamic System Simulation}

The dynamics of each chamber modelled with no inertial effects are governed by the ordinary differential equation in Equation (7), requiring only the volume as a state variable $(\underline{x}=[V])$. With the incorporation of inertial effects into the model, the flow (Q) is governed by the first order ordinary differential equation (ODE) of Equation (6). To run this model, both inflow and outflow rates must become additional state variables, adding two more $\operatorname{states}\left(\underline{x}=\left[V, Q_{1}, Q_{2}\right]\right)$. 


\subsection{The Valve Law}

In models where inertia is not included and the governing flow is defined by Equation (5), the valves are simulated simply by setting the flow rate to zero when the pressure gradient is positive. However, when inertial effects are included in the model, the flow rate $(\mathrm{Q})$ is governed by a first order ODE. In this case, setting the flow rate to zero will create a discontinuity, resulting in a physiologically inaccurate simulation. To solve this problem, the state vector is changed while solving, depending on the chamber state.

The PV diagram in Figure 4 shows the three state vectors ( $\underline{x})$ for a single cardiac chamber during the cardiac cycle. For example, during the transition from isovolumetric contraction to ejection, the system changes state from having just volume in the state vector $(\underline{x}=[V])$, to including exiting flow rate $\left(\underline{\mathrm{x}}=\left[\mathrm{V}, \mathrm{Q}_{2}\right]\right)$.

To change the state of the system, the state variables employed are triggered to change when either a flow rate or a pressure gradient becomes negative. When a flow rate $(\mathrm{Q})$ becomes negative, it is removed from the state vector to account for the valve closing in the absence of flow. Alternatively, when a pressure gradient becomes negative, the associated flow rate is reincluded in the state vector to account for the valve opening. Hence, the valve law for the model presented is "close on flow, open on pressure" where the valve opens on a negative pressure gradient, but is delayed from closing on a positive pressure gradient due to the inertia of the blood, matching normal physiological function [Opie, 1998]. 


\subsection{Ventricular Interaction}

When simulating ventricular interaction, the following steps are taken at each iteration using the current volumes $\left(V_{\mathrm{lv}}\right.$ and $\left.\mathrm{V}_{\mathrm{rv}}\right)$ to find their rates of change:

- Use Equation (16) to calculate the pressure in the pericardium from the total volume. $\left(\mathrm{V}_{\mathrm{tot}}=\mathrm{V}_{\mathrm{lv}}+\mathrm{V}_{\mathrm{rv}}\right)$

- Using Equations (1)-(3), (8) and (9) along with the equation for the pressure balance across the septum:

$$
\mathrm{P}_{\mathrm{spt}}\left(\mathrm{V}_{\mathrm{spt}}\right)=\mathrm{P}_{\mathrm{lvf}}\left(\mathrm{V}_{\mathrm{lvf}}\right)-\mathrm{P}_{\mathrm{rvf}}\left(\mathrm{V}_{\mathrm{rvf}}\right)
$$

the following equation can be numerically solved to find $V_{\text {spt }}$.

$$
\begin{aligned}
& e E_{\mathrm{es}, \mathrm{spt}}\left(\mathrm{V}_{\mathrm{spt}}-\mathrm{V}_{\mathrm{d}, \mathrm{spt}}\right)+(1-\mathrm{e}) \mathrm{P}_{\mathrm{o}, \mathrm{spt}}\left(\mathrm{e}^{\lambda_{\mathrm{spt}}\left(\mathrm{V}_{\mathrm{spt}}-\mathrm{V}_{\mathrm{o}, \mathrm{spt}}\right)}-1\right) \\
& =\mathrm{e} \mathrm{E}_{\mathrm{es}, \mathrm{lvf}}\left(\mathrm{V}_{\mathrm{lv}}-\mathrm{V}_{\mathrm{spt}}-\mathrm{V}_{\mathrm{d}, \mathrm{lvf}}\right)+(1-\mathrm{e}) \mathrm{P}_{\mathrm{o}, \mathrm{lvf}}\left(\mathrm{e}^{\lambda_{\mathrm{lvf}}\left(\mathrm{V}_{\mathrm{lv}}-\mathrm{V}_{\mathrm{spt}}-\mathrm{V}_{\mathrm{o}, \mathrm{lvf}}\right)}-1\right) \\
& -\mathrm{e} \mathrm{E}_{\mathrm{es}, \mathrm{rvf}}\left(\mathrm{V}_{\mathrm{rv}}+\mathrm{V}_{\mathrm{spt}}-\mathrm{V}_{\mathrm{d}, \mathrm{rvf}}\right)-(1-\mathrm{e}) \mathrm{P}_{\mathrm{o}, \mathrm{rvf}}\left(\mathrm{e}^{\lambda_{\mathrm{rvf}}\left(\mathrm{V}_{\mathrm{rv}}+\mathrm{V}_{\mathrm{spt}}-\mathrm{V}_{\mathrm{o}, \mathrm{rvf}}\right)}-1\right)
\end{aligned}
$$

- Given $\mathrm{V}_{\mathrm{spt}}, \mathrm{V}_{\mathrm{lvf}}$ and $\mathrm{V}_{\mathrm{rvf}}$, calculate $\mathrm{P}_{\mathrm{lvf}}$ and $\mathrm{P}_{\mathrm{rvf}} \mathrm{using}$ Equation (15).

- Use the values of $\mathrm{P}_{\mathrm{lvf}}, \mathrm{P}_{\mathrm{rvf}}$ and $\mathrm{P}_{\text {peri }}$ to calculate $\mathrm{P}_{\mathrm{lv}}$ and $\mathrm{P}_{\mathrm{rv}}$ using Equations (11) and (12).

- With $\mathrm{P}_{\mathrm{lv}}$ and $\mathrm{P}_{\mathrm{rv}}$, it is now possible to calculate flow rates, and thus, rates of change of ventricular volumes $\left(\mathrm{V}_{\mathrm{lv}}\right.$ and $\left.\mathrm{V}_{\mathrm{rv}}\right)$. 
The initial volumes are approximated based on normal heart function and the initial flow rates are calculated using Equation (5). Alternatively, the results from a previously run model can be used as the initial conditions for a new model.

\section{Model Verification}

The model is first verified to simulate basic CVS function as defined in Guyton and Hall (2001) in a stable and robust fashion. Then static response verification involves simulating changes in model parameters and comparing the resulting change in steady state dynamics with known physiological response from medical textbooks [Guyton and Hall, 2001]. Next, transient response verification is carried out simulating the dynamic response of the model to changes in model parameters and comparing the outputs with published experimental results [Slinker and Glantz, 1986, 1989]. Finally, model simulations of disease states are compared with known physiological response as published in medical textbooks [Braunwald et al., 2001; Parrillo and Bone, 1995].

\subsection{Verifying basic model function}

Simulations of the closed loop model show no dependence on initial conditions and produce stable results. Different initial conditions converged to the same steady state solution after a period of about two heartbeats. Long-term stability was verified over a period of 40 seconds, or about 66 heartbeats, with no divergence. 
The patient parameters used in this model are based on those found in the literature to produce model outputs comparable to an average human [Burkhoff and Tyberg, 1993; Chung et al., 1997]. Figure 6 shows the simulated output pressures and volumes, which compare well with target performance metrics from Guyton and Hall (2001). A typical ventricle stroke volume (SV) is about $60-70 \mathrm{ml}$, and is marked on the graph. The aortic pressure in an average person is around $120 / 80 \mathrm{mmHg}$ as marked (ao), while a normal pulmonary artery pressure is about $25 / 8 \mathrm{mmHg}$ as marked (pa). The return pressure entering the right heart is typically around $0 \mathrm{mmHg}$, but it can be negative since the pressure in the thoracic cavity is generally about $-4 \mathrm{mmHg}$. The return pressure to the left ventricle is typically $1-5 \mathrm{mmHg}$. All of the magnitudes reported are comparable to expected values verifying the model assumptions and solution.

The model is also found to simulate ventricular interaction effectively. Figure 7 plots the deflection of the septum wall, the pericardium and the left and right ventricle volumes during the cardiac cycle. The plot shows how this minimal approach to simulating ventricular interaction includes the effects of septal deflection and changes in volume of the pericardium.

The next stage of this research is to verify that the model captures known physiological trends in CVS dynamics. Model verification is divided into three sections including static response verification, transient response verification and simulation of disease state. Static response verification is commonly used throughout the literature to verify CVS models [Chung et al., 1997; Santamore and Burkhoff, 1991; Olansen et al., 2000]. Parameters in the model are altered, such as the thoracic cavity pressure, and the change in 
steady state dynamics of the model are investigated. The ability of models to simulate transient CVS dynamics is much less common in the literature. Models parameters are adjusted and the transient response of the model, immediately after these alterations, is investigated. Finally, simulation of disease state tests the ability of the model to simulate CVS dysfunctions and produce outputs that match known physiological trends.

\subsection{Static Response Verification}

Normal thoracic cavity pressure $\left(\mathrm{P}_{\mathrm{th}}\right)$ in humans averages about $-4 \mathrm{mmHg}$. However, if this pressure is increased, as occurs with a patient on a respirator during positive pressure breathing, cardiac output (CO) decreases [Guyton and Hall, 2001]. Figure 8 shows the model output for both $0 \mathrm{mmHg}$ and $-4 \mathrm{mmHg}$ thoracic pressure on a PV diagram. The diagram shows the stroke volume, and thus the cardiac output, is decreased by $5 \%$ with the increased thoracic pressure, matching known physiological response.

A second example shows the effect of changing the systemic circulatory resistance. Figure 9 shows that as systemic resistance is increased, stroke volume and thus cardiac output, decreases. The results also show an increase in aortic pressure from $120 / 80 \mathrm{mmHg}$ to $160 / 130 \mathrm{mmHg}$ when the systemic resistance is doubled. These results match the usual rise in blood pressure and drop in cardiac output associated with increased resistance due to arteries becoming blocked [Guyton and Hall, 2001].

These static verification results show the potential of this model for accurately simulating trends in the cardiovascular system in response to changes 
in therapy or physiology. However, as with most other models in the literature, these results only prove the ability of the model to capture steady state responses.

\subsection{Transient Response Verification}

The transient response of the minimal model was verified against experimental results for both ventricular interaction and cardiopulmonary interaction [Smith, 2004]. A brief example is shown in this paper that illustrates the ability of the model to capture transient dynamics in CVS function during ventricular interaction. This example references experimental research carried out on canines by Slinker and Glantz (1989) investigating ventricular interaction.

Experimental data found in the literature usually involved surgically removing the heart from the circulation system and investigating static responses [Maughan et al., 1987; Weber et al., 1981; Glantz and Parmley, 1978; Santamore and Dell'Italia, 1998]. Fluid is injected into one side of the heart, and the effect on the other side of the heart is investigated. Slinker and Glantz (1989) carried out research on anaesthetized dogs with the heart still connected to the CVS. Their aim was to investigate transient responses due to ventricular interaction.

The procedure involved sequentially constricting and releasing the pulmonary artery and vena-cava, as labelled in Figure 1. Pulmonary artery constriction (PAC) causes an increase in resistance downstream of the right ventricle, increasing the pressure against which the right ventricle must pump. 
Applying vena-cava constriction (VCC) caused a higher resistance upstream of the right ventricle, decreasing the right ventricle filling pressure. When the constrictions are subsequently released the CVS responds again, settling to its original state.

Volumes, pressures and flow rates around both the right and left ventricles were measured to determine the response of the CVS to these changes in state. The constrictions and their subsequent releases directly affect the function of the right ventricle, and affect left ventricle function though ventricular interaction. Thus, by investigating the response of the left ventricle, Slinker and Glantz (1986) were able to comment on the relative effects of direct and series interaction on CVS dynamics. Direct interaction occurs due to the septum and the pericardium. Series interaction results from the connection between the outlet of each ventricle and the inlet of the other. The minimal model can account for both types of interaction.

Figure 10(a) reproduces the experimentally measured variation in left ventricle end-diastolic area, which is assumed to be proportional to left ventricle end-diastolic volume. The black dots are experimentally measured data. The solid and dashed lines plot the results of a statistical analysis by Slinker and Glantz (1986) to capture the changes, and are not experimental data. Figure 10(b) plots the variation in simulated left ventricle volume $\left(\mathrm{V}_{\mathrm{lv}}\right)$ using the CVS minimal model. The dark line along the top of the graph marks the variation in end-diastolic volume. The model results in Figure 10(b) are seen to contain all the major trends and transient dynamics in the experimental results of Figure 10(a).

This example illustrates the transient verification approach taken to val- 
idating the ventricular interaction in the minimal model. The results verify that the model is capable of capturing the transient dynamic response of the CVS due to both direct and series ventricular interaction. No other model found in the literature has been verified to capture these transient responses in CVS dynamics due to ventricular interaction. Using experimental methods to investigate the effects of CVS function on ventricle dynamics is difficult. However, the minimal model enables much more detailed analysis of the individual contributions and dynamics to be fully studied, despite the relatively simple, minimal model structure.

\subsection{Simulating Disease State}

The minimal model developed is specifically aimed to be of direct use to medical staff to improve understanding of the affect of disease state on CVS function and assist in diagnosis. The model was found to simulate a variety of disease states [Smith, 2004]. This section illustrates an example where the effects of a myocardial infarction, which can result in a heart attack, is simulated.

Myocardial infarction occurs where part of the heart muscle has died due to lack of oxygen and can lead to catastrophic heart failure. The main impact of myocardial infarction is a drop in ventricle contractility because the weakened heart is no longer able to eject an adequate amount of blood. Decreased contractility is simulated in the minimal model by decreasing the parameter $\mathrm{E}_{\mathrm{es}}$ in Equation (1), which decreases the slope of the ESPVR. Characteristic haemodynamics of decreased contractility include increased 
ventricle filling pressure, a rise in ventricle volume, a drop in stroke volume, and decreased systemic pressure [Kumar and Parrillo, 1995; Braunwald et al., 2001; Parrillo and Bone, 1995].

The PV diagram in Figure 11 shows the simulated effect of halving contractility $\left(0.5 \times \mathrm{E}_{\mathrm{es}, \mathrm{lvf}}\right)$ on a PV diagram. The drop in contractility causes a shift in the cardiac cycle to the right, increasing the ventricle volume. This shift also causes a rise in the diastolic pressure resulting in an increase in ventricle filling pressure. Figure 11 also shows the decrease in stroke volume, resulting in a drop in cardiac output, that matches the listed known physiological trends. The model results also show how decreased contractility causes a drop in end-systolic pressure.

If a patient is found to have symptoms of reduced contractility, a vasodilator is often administered to reduce peripheral resistance. The vasodilator causes a recovery in cardiac output, but at the expense of a drop in blood pressure. The effect of administering a vasodilator is also simulated by halving the systemic vascular resistance $\left(0.5 \times \mathrm{R}_{\mathrm{sys}}\right)$, shown as the shaded region in Figure 11. The model captures the increase in stroke volume and decrease in end-systolic pressure that occurs as a result of vasodilator administration. This example highlights the ability of the model to capture disease state and therefore assist medical staff in choosing suitable therapies for different types of cardiac dysfunction by simulating the known effect of a drug on CVS dynamics. 


\subsection{Summary of Model Verification}

Examples of both static and transient model verification have been shown. While static verification is commonly seen in the literature, there are very few examples of verifying a model against transient CVS dynamics. Transient verification illustrates the diagnostic and therapeutic potential of CVS models that are proven to capture dynamic trends in CVS function. As shown, the minimal model concept enables CVS models to improve understanding in a much wider range of CVS function.

The verification carried out here and in Smith (2004) validates the model can simulate commonly accepted physiological trends. These preliminary results show the potential of the model for use as a decision support tool. More rigorous testing involving statistical analysis needs to be carried out to prove the usefulness of the model in the clinical environment.

\section{Conclusion}

The minimal model approach presented for modelling the CVS is shown to offer numerical stability and robustness. A means of modelling the valve dynamics is presented that accommodates inertial effects, and avoids discontinuities and numerical instability while providing physiologically realistic performance. The closed loop system model was shown to accurately capture basic trends in human cardiovascular system function. The static, and uniquely, the transient response of the model is verified against experimental results. No other example of transient verification of a CVS model for ventricular interaction was found in the literature. Finally, an example is 
given illustrating the potential of the model for accurately simulating disease state. The overall results show the significant potential of this approach to modelling the CVS system and the ability of this simple model to capture a wide range of unexpectedly rich dynamic behaviours.

\section{References}

Association, A. H. (2004). Cardiovascular disease statistics.

Beyar, R., Hausknecht, M. J., Halperin, H. R., Yin, F. C., and Weisfeldt, M. L. (1987). Interaction between cardiac chambers and thoracic pressure in intact circulation. Am. J. Physiol., 22(253):H1240-H1252.

Braunwald, E., Zipes, D. P., and Libby, P. (2001). Heart Disease, A text book of cardiovascular medicine. W.B. Saunders Company, Philadelphia, 6 edition.

Burkhoff, D. and Tyberg, J. V. (1993). Why does pulmonary venous pressure rise after onset of lv dysfunction: a theoretical analysis. Am. J. Physiol., 265:H1819-H1828.

Chung, D., Niranjan, S., Clark, J., Bidani, A., and Johnston, W. (1997). A dynamic model of ventricular interaction and pericardial influence. Am. J. Physiol., 14(272):H2924-H2962.

Fung, Y. (1990). Biomechanics: Motion, flow, stress, and growth. SpringerVerlag, San Deigo. 
Glantz, S. A. and Parmley, W. W. (1978). Factors which affect the diastolic pressure-volume curve. Circ Res, 42(2):171-80.

Guyton, A. C. and Hall, J. E. (2001). Textbook of medical physiology. W.B. Saunders Company, Philadelphia, 10 edition.

Kumar, A. and Parrillo, J. E. (1995). Shock: Classification, pathophysiology, and approach to management. In Parrillo, J. E. and Bone, R. C., editors, Critical Care Medicine, Principles of diagnosis and management. Mosby, St. Louis, Missouri.

Legrice, I., Hunter, P., and Smaill, B. (1997). Laminar structure of the heart: a mathematical model. Am J Physiol, 272:H2466-76.

Maughan, W. L., Sunagawa, K., and Sagawa, K. (1987). Ventricular systolic interdependence: volume elastance model in isolated canine hearts. Am. J. Physiol., 253:H1381-H1390. [Ventricular interaction].

Melchior, F. M., Sriniwasan, R. S., and Charles, J. B. (1992). Mathematical modeling of human cardiovascular system for simulation of orthostatic response. Am. J. Physiol., 262:H1920-H1933.

Olansen, J., Clark, J., Khoury, D., Ghorbel, F., and Bidani, A. (2000). A closed-loop model of the canine cardiovascular system that includes ventricular interaction. Comp. Biomed. Res., 33:260-295.

Opie, L. H. (1998). The heart : physiology, from cell to circulation, 3rd ed. Lippincott-Raven, Philadelphia. 
Parrillo, J. E. and Bone, R. C. (1995). Critical Care Medicine, Principles of diagnosis and management. Mosby, St. Louis, Missouri.

Peskin, C. S. and McQueen, D. M. (1992). Cardiac fluid dynamics. Crit Rev Biomed Eng, 20(5-6):451-9.

Santamore, W. P. and Burkhoff, D. (1991). Hemodynamic consequences of ventricular interaction as assessed by model analysis. Am. J. Physiol., 260:H146-H157.

Santamore, W. P. and Dell'Italia, L. J. (Jan/Feb 1998). Ventricular interdependence: Significant left ventricular contributions to right ventricular systolic function. Prog. Cardiovascular Diseases, 40(4):289-308.

Slinker, B. K. and Glantz, S. A. (1986). End-systolic and end-diastolic ventricular interaction. Am J Physiol, 251(5 Pt 2):H1062-75.

Slinker, B. K. and Glantz, S. A. (1989). Beat-to-beat regulation of left ventricular function in the intact cardiovascular system. Am J Physiol, 256(4 Pt 2):R962-75.

Smith, B., Chase, J., Nokes, R., Shaw, G., and David, T. (2003). Velocity profile method for time varying resistance in minimal cvs models. Physics in Medicine and Biology, 48(20):3375 - 3387.

Smith, B. W. (2004). Minimal Haemodynamic Modelling of the Heart and Circulation for Clinical Application. PhD thesis, Canterbury University.

Tsitlik, J. E., Halperin, H. R., Popel, A. S., Shoukas, A. A., Yin, F. C., and Westerhof, N. (1992). Modeling the circulation with three-terminal 
electrical networks containing special nonlinear capacitors. Ann Biomed Eng, 20(6):595-616.

Weber, K. T., Janicki, J. S., Shroff, S., and Fishman, A. P. (1981). Contractile mechanics and interaction of the right and left ventricles. $A m J$ Cardiol, 47(3):686-95. 


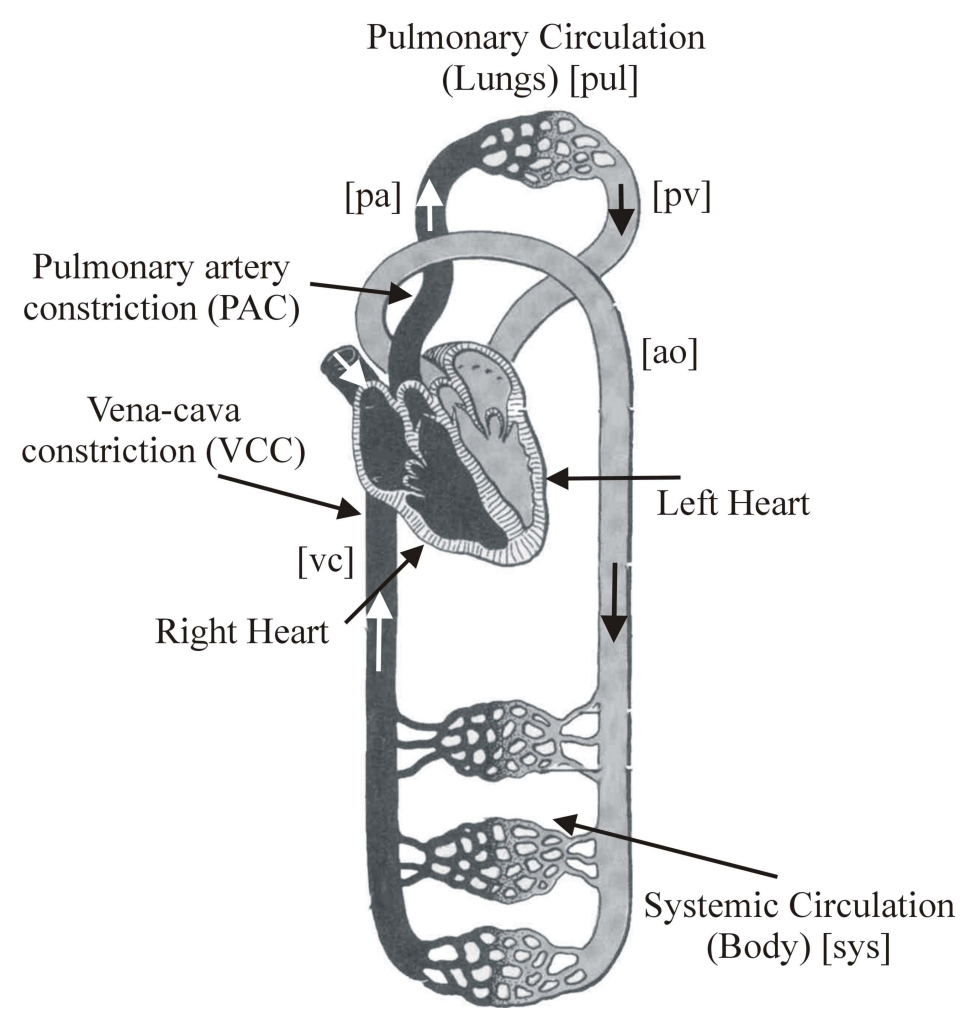

Figure 1: Diagram of the circulation system from Guyton and Hall (2001), with locations of the constrictions carried out by Slinker and Glantz (1986) labelled. 


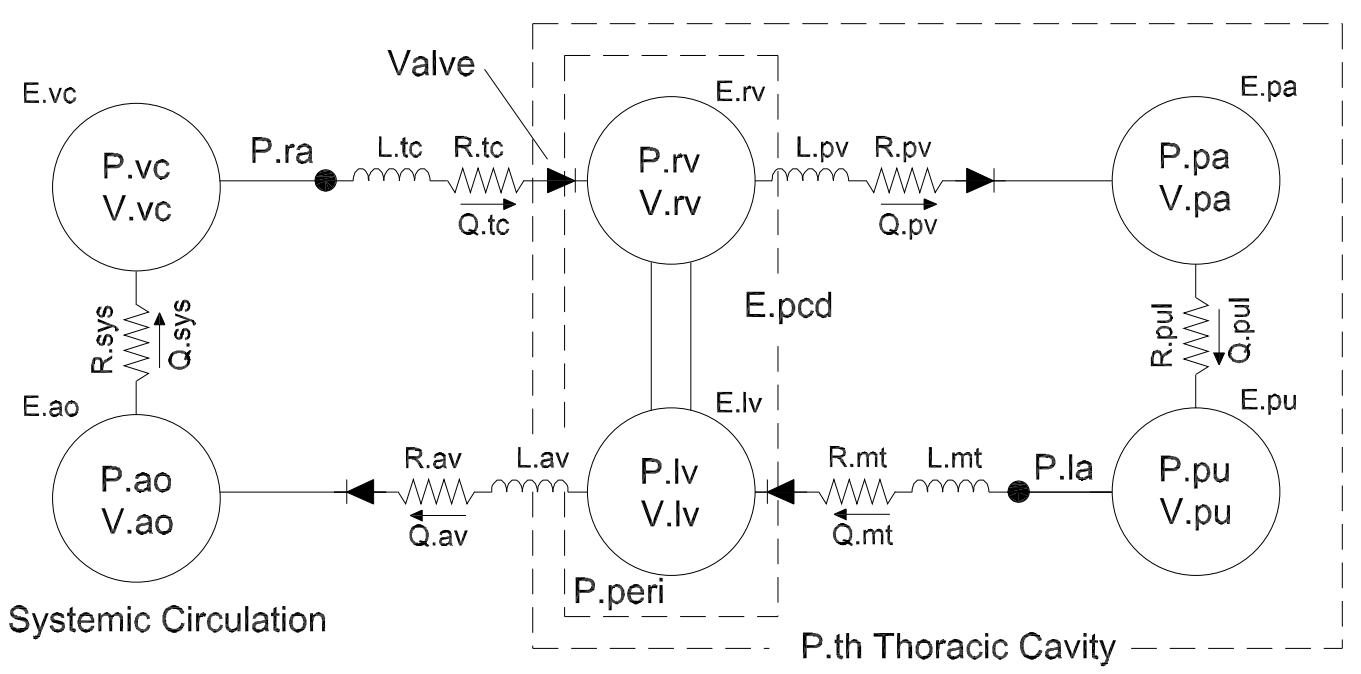

Figure 2: The presented closed loop model of the cardiovascular system.

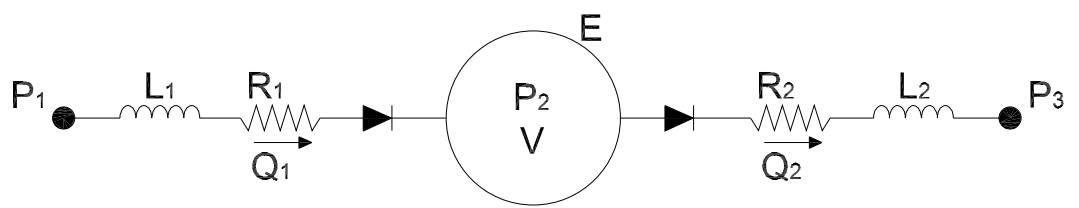

Figure 3: Single chamber model. 


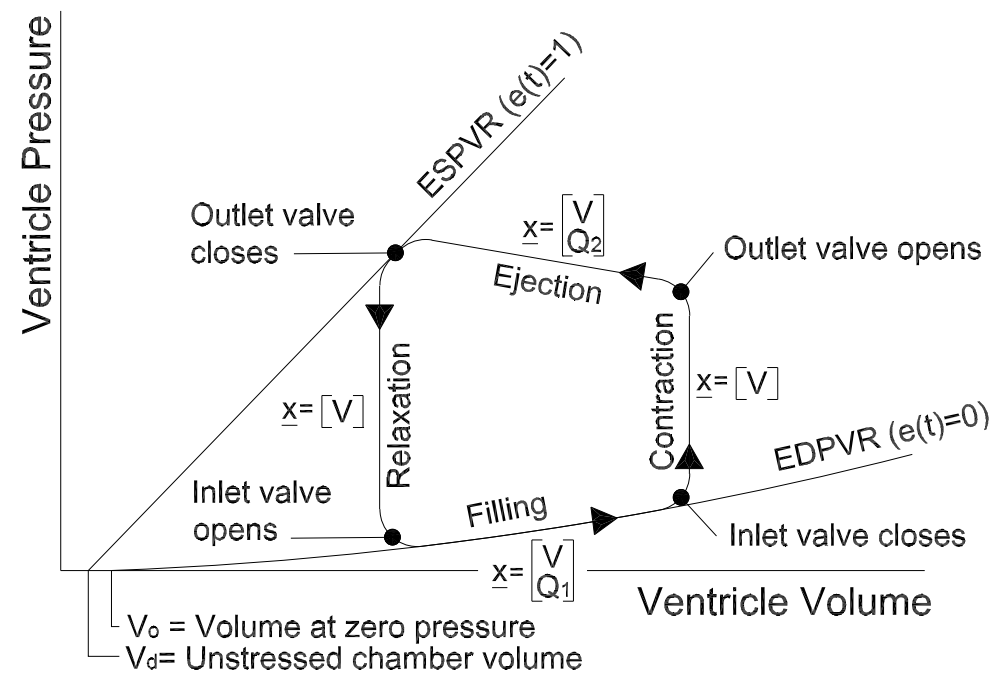

Figure 4: Pressure-volume diagram of the cardiac cycle and the variations in end-diastolic and end-systolic pressure-volume relationships. 


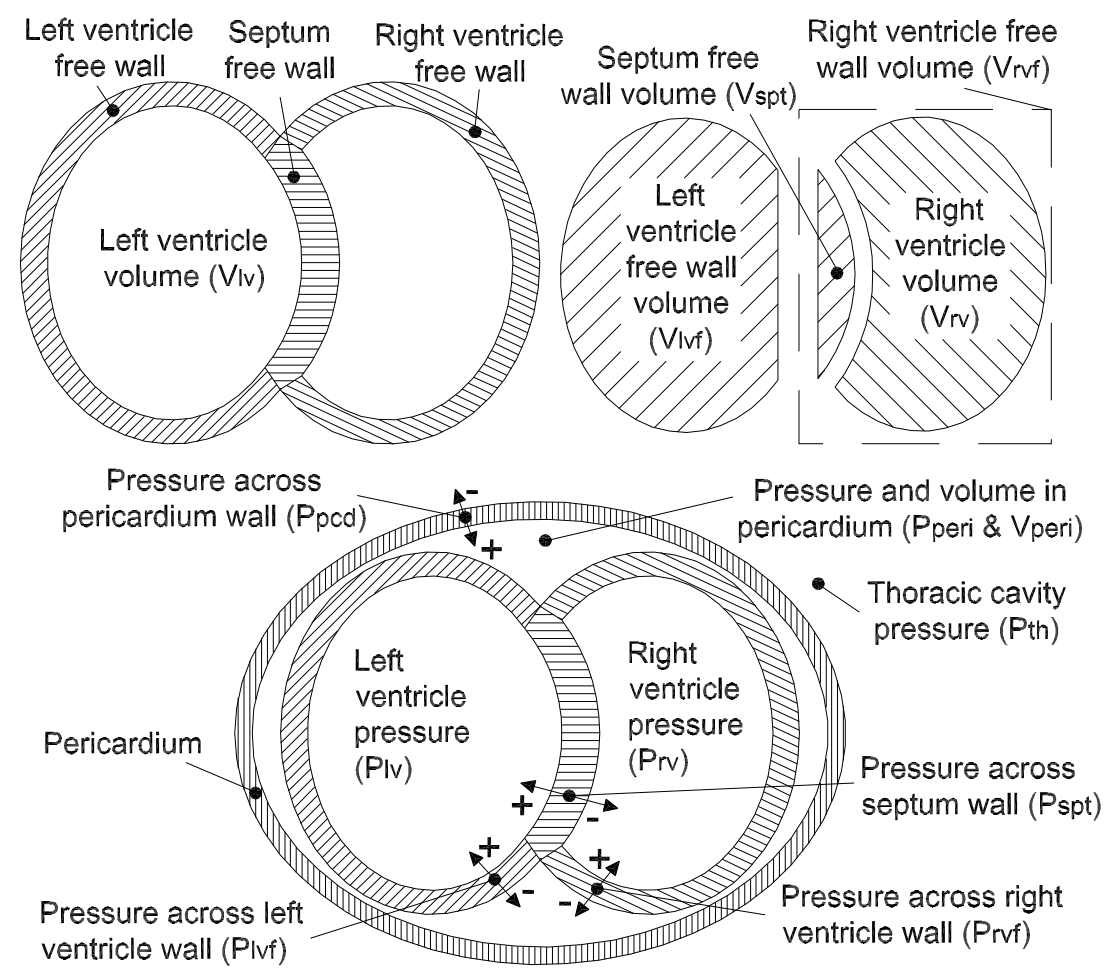

Figure 5: Septum, pericardium, left and right ventricle, and free wall volumes and pressures of the heart. 

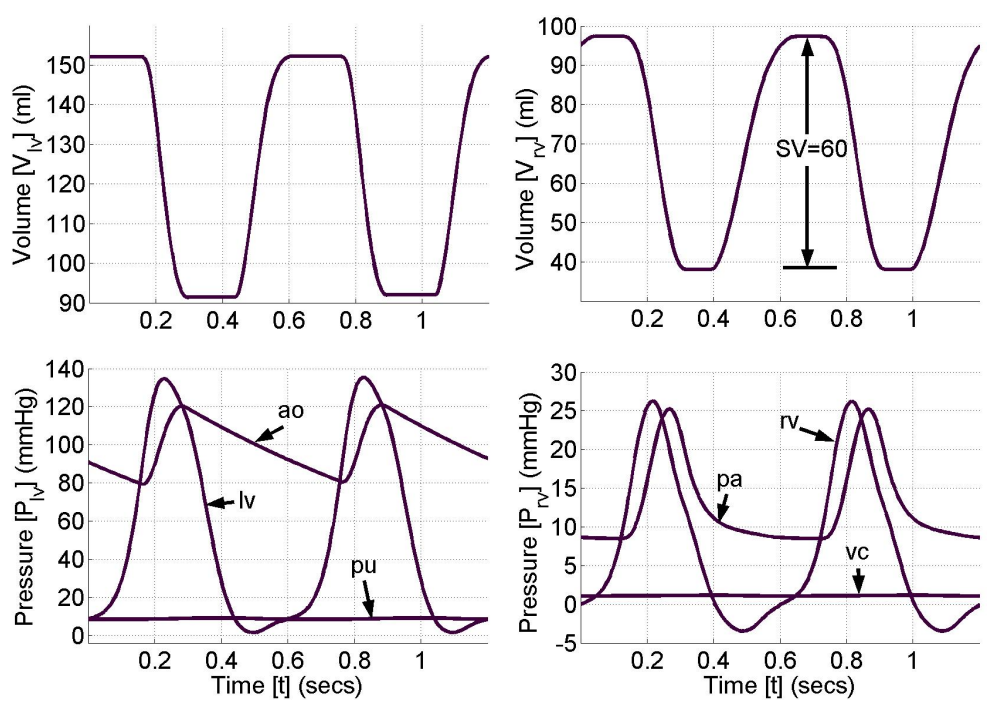

Figure 6: Simulation results for closed loop model with inertia and ventricular interaction.
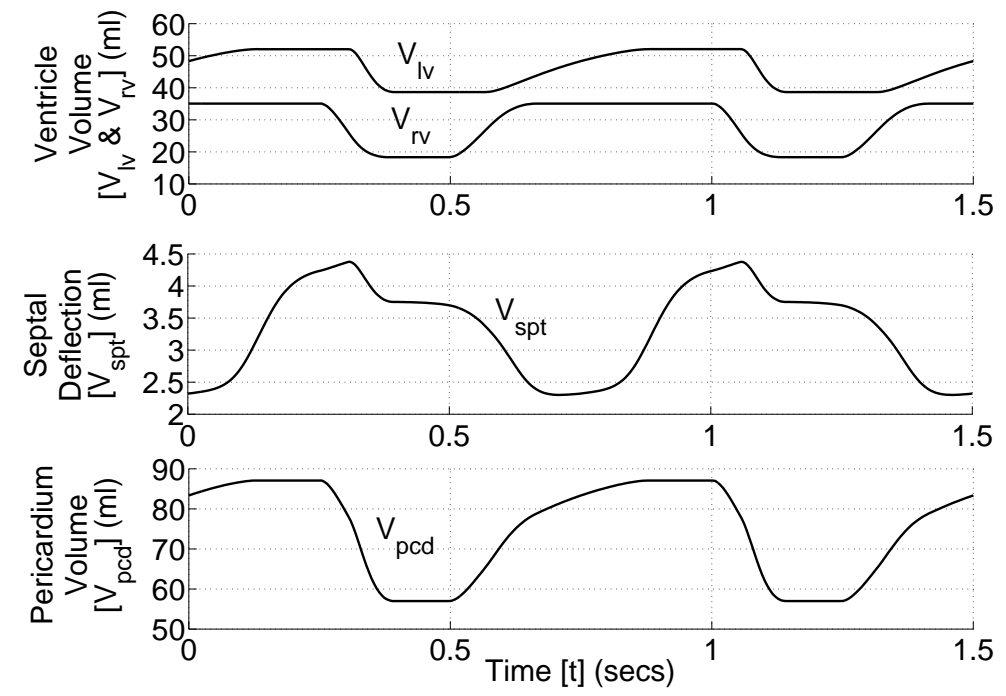

Figure 7: Comparing the variations in left and right ventricle volumes $\left(\mathrm{V}_{\mathrm{lv}}\right.$ and $\left.\mathrm{V}_{\mathrm{rv}}\right)$ with the septum deflection $\left(\mathrm{V}_{\mathrm{spt}}\right)$ and the pericardium deflection $\left(\mathrm{V}_{\mathrm{pcd}}\right)$. 

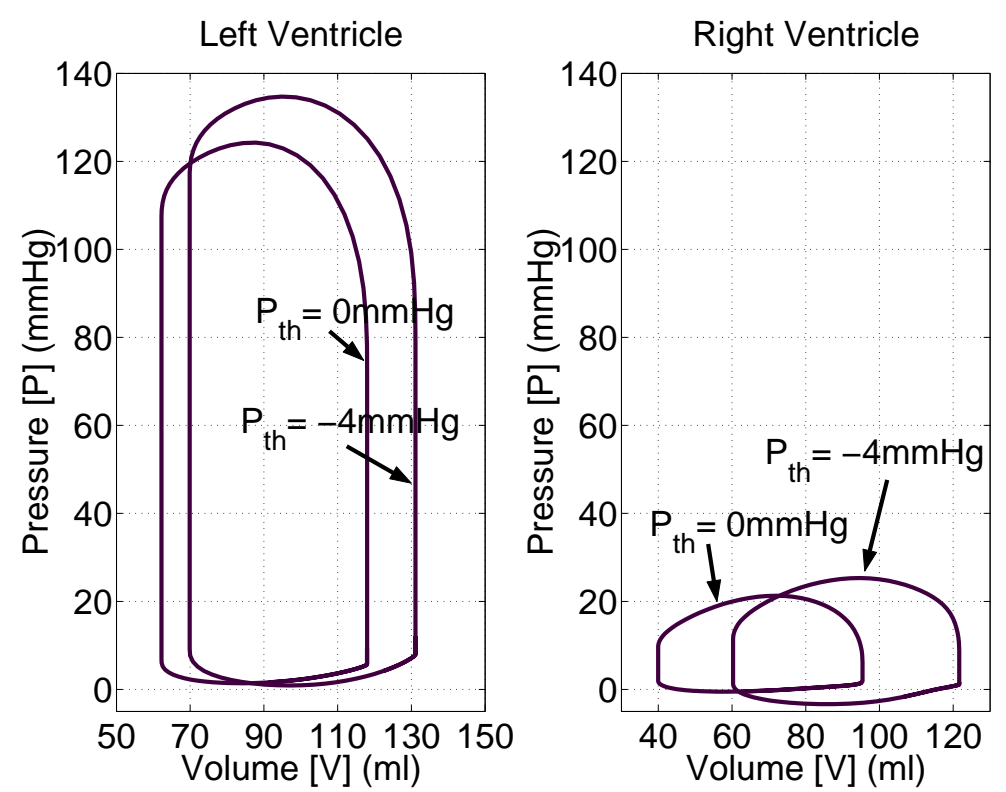

Figure 8: PV profiles for closed loop model with normal thoracic pressure $\left(\mathrm{P}_{\mathrm{th}}=-4 \mathrm{mmHg}\right)$, and high thoracic pressure $\left(\mathrm{P}_{\mathrm{th}}=0 \mathrm{mmHg}\right)$.
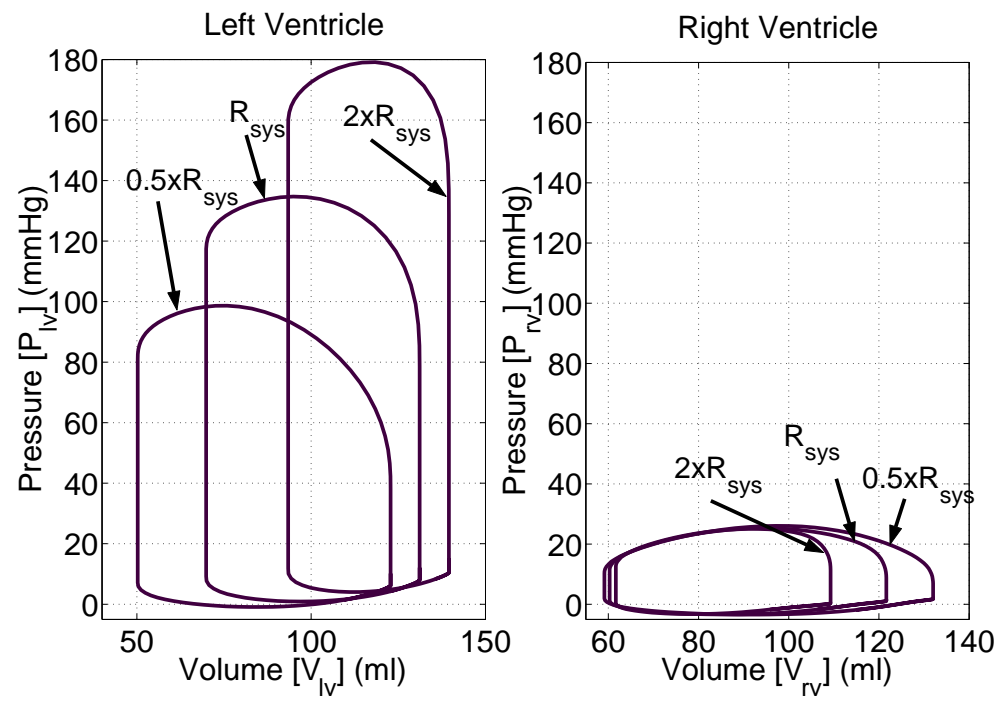

Figure 9: Simulation of changes in systemic resistance $\left(R_{\text {sys }}\right)$. 


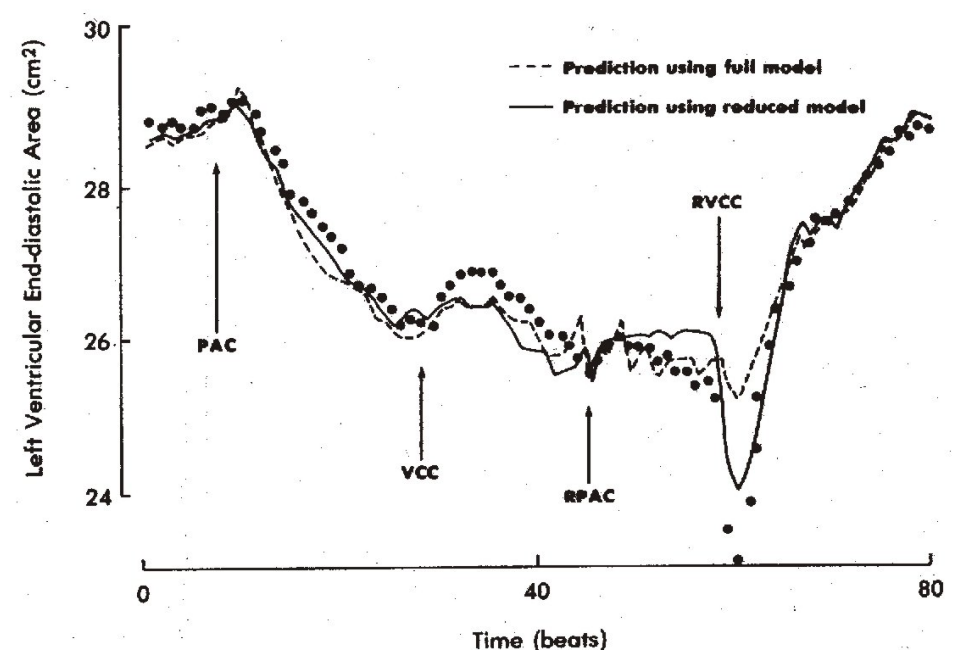

(a) Experimentally determined change in left ventricle enddiastolic area [Slinker and Glantz, 1986].

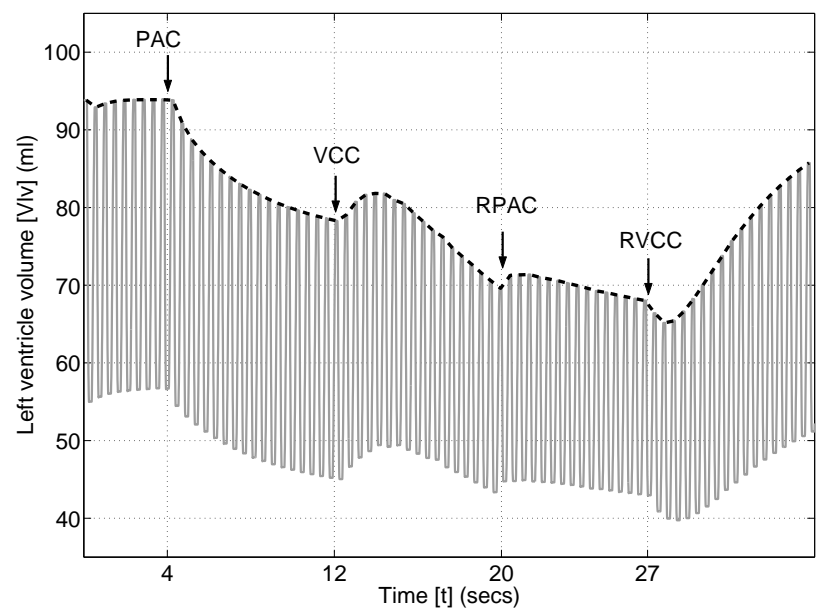

(b) Simulated variations in left ventricle volume (light grey) and end-diastolic volume (dark dashed line)

Figure 10: Comparing experimental and simulated changes for primary experiment. 


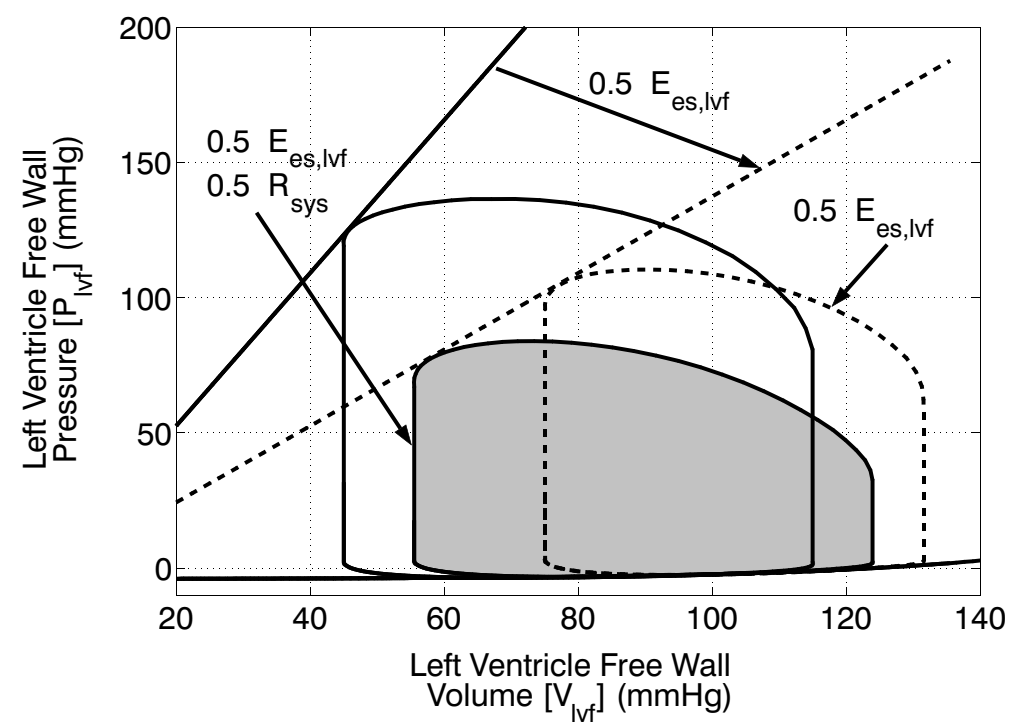

Figure 11: Minimal model simulated effect of decreased left ventricle contractility (dashed line) and decreased resistance simulating administration of a vasodilator (shaded area). 